http://jmscr.igmpublication.org/home/ ISSN (e)-2347-176x ISSN (p) 2455-0450

crossref DOI: https://dx.doi.org/10.18535/jmscr/v8i9.50

\title{
Wilsons Disease - A Varied Histological Spectrum
}

\author{
Authors \\ Dr Milap Shan MD Pathology, Dr M. Srividya DNB Pathology, \\ Dr Sindhu Kotla MD Pathology, Dr G. Archana MD Pathology
}

\begin{abstract}
Wilsons disease is an autosomal recessive disorder of copper metabolism that mostly presents with hepatic dysfunction followed by neurologic/psychiatric symptoms and hematological problems. Though the defect is already present at birth it rarely manifests before 5 years of age. Here we are presenting four cases of wilsons disease presenting with variable histopathological presentations at our hospital during the year 2020.
\end{abstract}

\section{Introduction}

Wilson disease is an autosomal recessive disease resulting from mutation in ATP7B gene, which is responsible for impaired copper excretion in bile and failure to incorporate copper into ceruloplasmin. This disorder is marked by accumulation of toxic levels of copper in liver, brain and eye.

Normally 40-60\% of ingested copper is absorbed in duodenum and proximal small intestine, from where it is transported and complexed with albumin and histidine to liver. Here free copper dissociates and is taken up by hepatocytes and is incorporated into enzymes and alfa2-globulin (apoceruloplasmim) to form ceruloplasmin which is secreted into the blood. Ceruloplasmin carries 90-95\% of copper in blood. Circulating ceruloplasmin is desialylated in liver and free copper is excreted in the bile, which is the primary route of copper elimination.

ATP7B gene located on chromosome 13, encodes a transmembrane copper transporting ATPase, that is expressed in hepatocyte canalicular membrane. Majority of patients are compound heterozygous with different loss of function mutations, affecting each ATP7B allele. The frequency of mutated allele is 1:100 and the prevalence of disease previously estimated as $1: 30,000$, but recent data suggested genetic prevalence of 1:7000. ${ }^{1,2}$ Loss of function mutation impairs incorporation of copper into ceruloplasmin and transport of copper into bile, leading to accumulation of copper in liver and decrease in plasma ceruloplasmin.

Copper deposited in liver causes liver injury by producing free reactive oxygen species by fenton reaction. Also this copper is released into blood causing hemolysis and alloing copper to accumulate in other organs such as brain, eye, kidney, joints and parathyroid gland. ${ }^{3}$

In genral, the upper age limit for considering Wilson disease is 40 years and the lower age limit is 5 years, although the disorder has been detected in children younger than 3 years and in adults older than 70 years. ${ }^{4}$ 
Wilsons disease has a range of clinical manifestations, from asymptomatic state to fulminant hepatic failure, chronic liver disease with or without cirrhosis, neurologic and psychiatric manifestations. $^{5}$

Three major patterns of hepatic involvement are as follows:

a) Chronic active hepatitis b)cirrhosis c) fulminant hepatic failure.

On histopathological examination there may be mild to moderate fatty change (steatosis) associated with focal hepatocyte necrosis. Acute fulminant hepatitis may mimic acute viral hepatitis. chronic hepatitis in Wilson disease exhibits moderate to severe inflammation and hepatocyte necrosis,areas of fatty change and features of steatohepatitis. In advanced cases cirrhosis may be seen.

Copper deposition in hepatocytes can be demonstrated by special stains - rhodamine stain for copper and orcein stain for copper associated protein. However in core biopsies it is not alwys possible to demonstrate copper due to variable stages of disease.

\section{Case-1}

A 14 year male patient presented with jaundice in the opd, for which he was evaluated in which liver function test (LFT) showed elevated total bilirubin, SGOT, SGPT, GGT and globulins.

Prothrombin time was also elevated. Serum ceruloplasmin- 9.3ng/dl, serum copper-142.2ng/dl and 24 hour urine copper- 720ug. On ultrasonography there was coarse echotecture of liver suggestive of liver parenchymal disease, mild edematous mural thickening of gall bladder and splenomegaly. Further a core biopsy of liver was submitted to histopathology, which demonstrated a chronic hepatitis pattern of injury with bridging fibrosis along with perivennular and focal pericellular fibrosis. Orcein stain shows patchy intra-cytoplasmic granular staining of copper. Negative for viral serology and autoimmune profile.
Chronic hepatitis with bridging fibrosis.

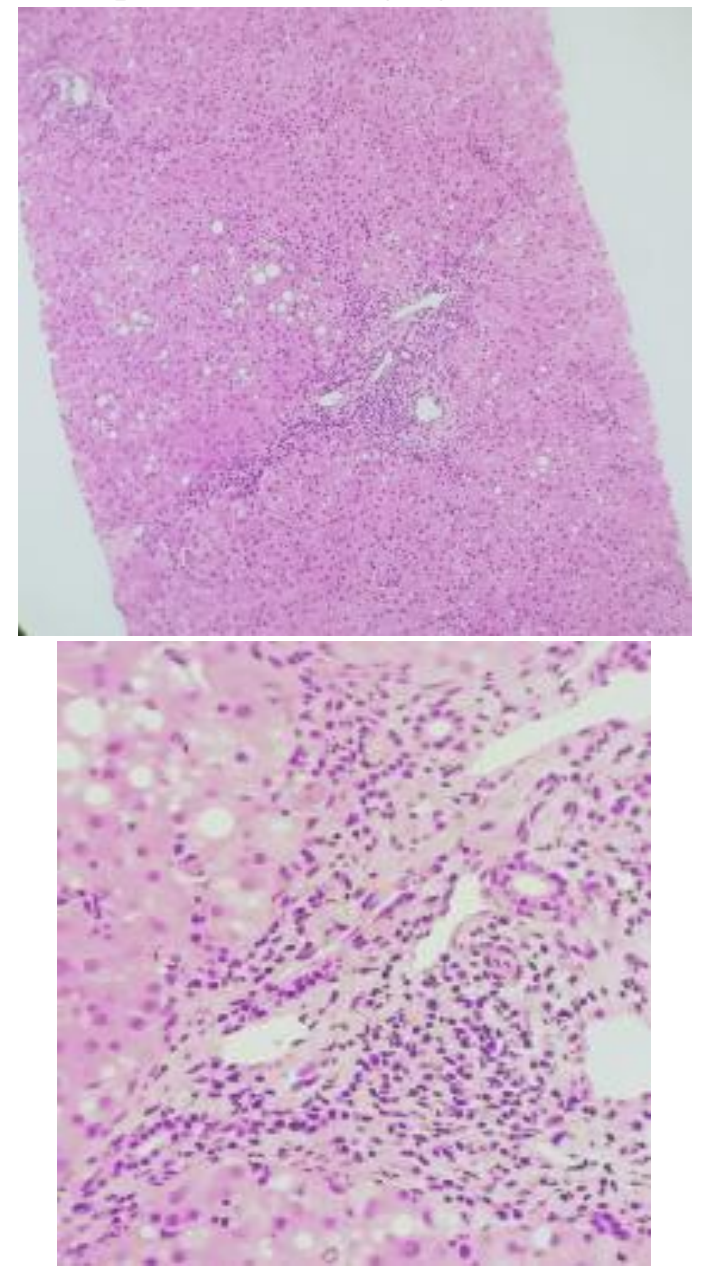

\section{Case-2}

A 6 year old male patient who was asymptomatic with a known history of wilsons disease in sibling. $\mathrm{He}$ was evaluated for liver function test which showed elevated SGOT, SGPT, GGT and prolonged prothrombin time. Serum ceruloplasmin $-12.5 \mathrm{ng} / \mathrm{dl}$.

Negative for viral markers and autoimmune profile.

A liver core biopsy was submitted for histopathological examination which demonstrated steatohepatitic pattern of injury with bridging fibrosis and incomplete nodule formation with copper retention as highlighted by rhodamine stain. Considering low serum ceruloplasmin and serum copper favoured diagnosis of wilsons disease. 
Steatohepatitic pattern of injury with bridging fibrosis.

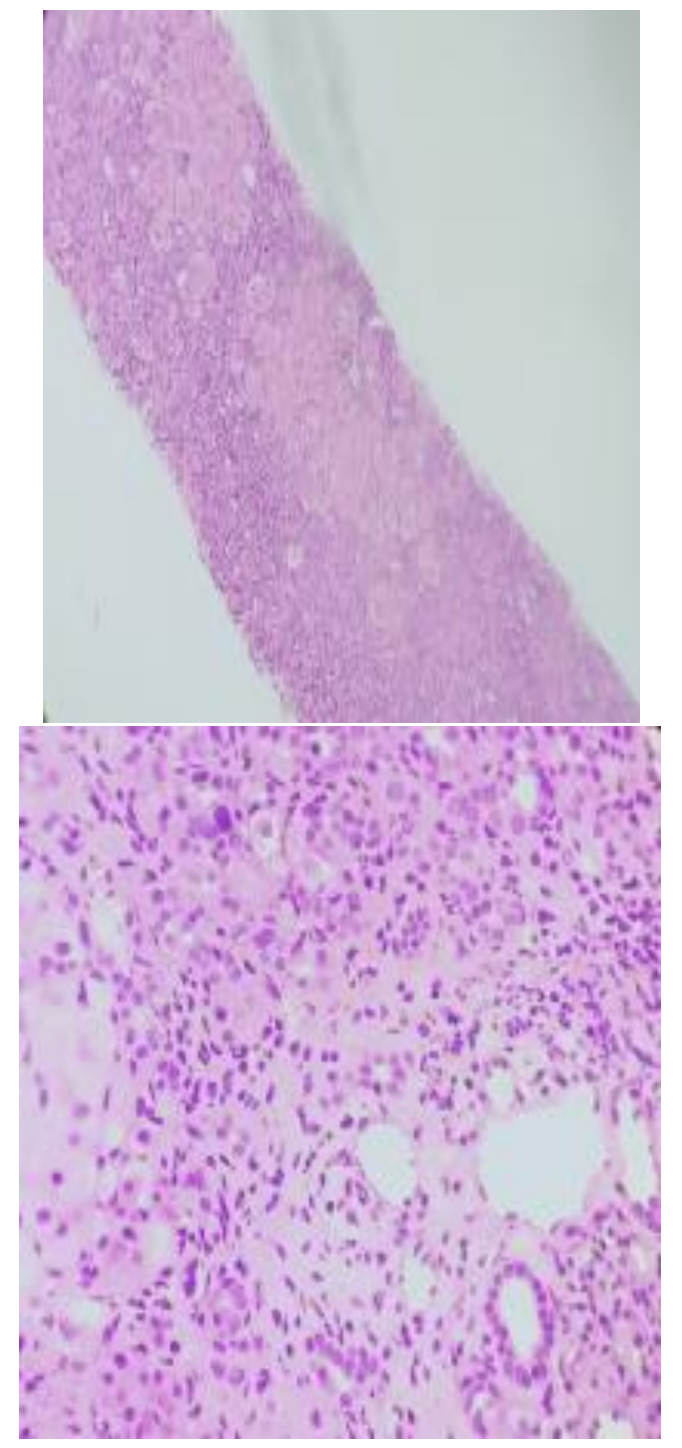

\section{Case-3}

A 8 year old male child presented with jaundice in opd, for which he was evaluated. Liver function test showed elevated total bilirubin, SGOT, SGPT, alkaline phosphatase, globulin and prothrombin time. Serum ceruloplasmin- $16.59 \mathrm{ng} / \mathrm{dl}$. Negative for viral markers and autoimmune profile. A liver core biopsy was submitted for histopathological examination, which showed hepatitis pattern of injury with bridging and confluent necrosis with parenchymal collapse and regenerating liver nodules. Swollen hepatocytes, glycogenated nuclei with small droplet type steatosis along with positive staining for copper and copper associated protein suggest diagnosis of Wilson disease.
Hepatocytes with glycogenated nuclei

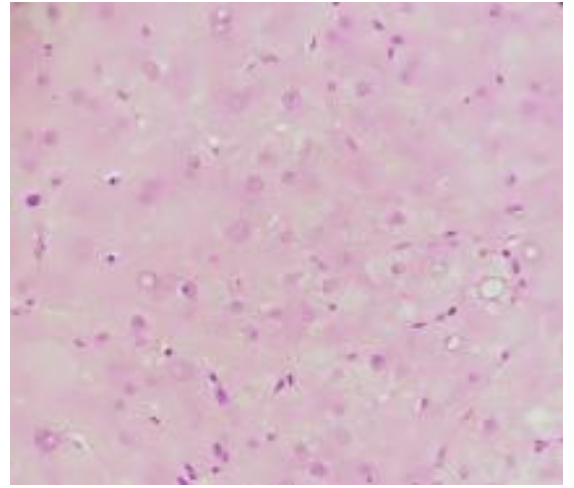

\section{Case-4}

A 10 year male chid presented with acute liver failure with elevated total bilirubin, SGOT, SGPT and globulin. Seumceruloplasmin $-31.3 \mathrm{ng} / \mathrm{dl}$. Negative for viral markers and autoimmune profile. Patient underwent liver transplantation. Liver explant on histopathology showed confluent multilobar/ pan acinar massive hepatic necrosis , consistant with diagnosis of acute liver failure. Tissue copper estimation was adviced.

Confluent / panacinar massive hepatic necrosis

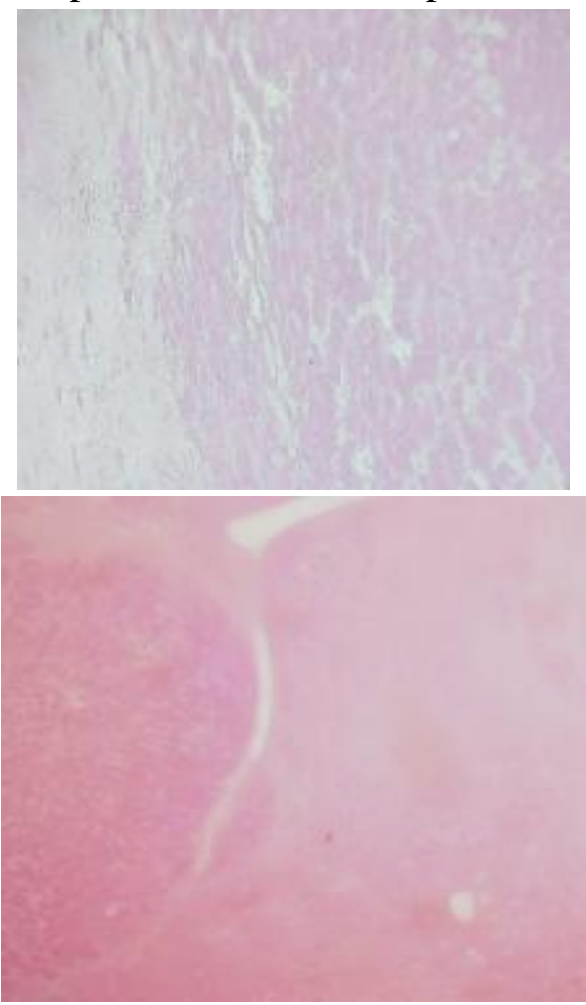


Copper demonstration in hepatocytes

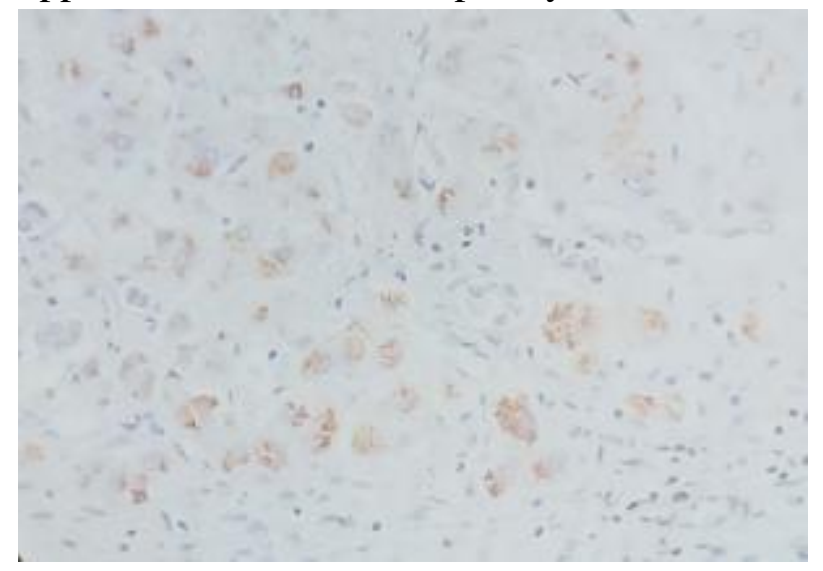

\section{Discussion}

Wilsons disease presents with a combination of liver disease, neurologic symptoms and kayserfleischer corneal ring. Patients who majorly present with liver disease do not demonstrate kayser-fleischer ring ${ }^{6}$.

Histopathology of wilsons disease is highly variable ranging from mild to severe chronic hepatitis, from simple steatosis to prominent steatohepatitis and showing confluent necrosis in fulminant cases. On progressing fibrosis and macronodular cirrhosis are observed. Glycogenated nuclei, portal inflammation and steatosis are almost always present. Copper demonstration by rhodamine stain does not rule out the diagnosis and is not always found. ${ }^{7,8}$

A study conducted by Vieira Barbosa joana et al in ten patients during the year 2004-2016 showed varied histological spectrum. Five patents showed mild to moderate chronic hepatitis, three patients presented with cirrhosis, two patients showed macrovesicular steatosis with steatohepatitis along with mild fibrosis and last one patient had a pattern of multifocal necrosis on a background of fibrosis 9 .

In our cases one patient showed chronic hepatitis, second one showed steatohepatitis, third one showed hepatitis pattern of injury with glycogenated nuclei and fourth one showed massive liver necrosis mimicking autoimmune hepatitis leading to acute liver failure.

Due to its highly variable presentation any patient with unclear hepatic or neurologic disease wilsons disease should be considered as differential diagnosis.

Diagnosis of Wilson disease is not straightforward, it should be suspected in combination of clinical features, family history, laboratory tests and confirmed by intrahepatic copper determination and genetic analysis.

\section{Conclusion}

Wilsons disease is a rare and highly fatal disease which should be always included in the differential diagnosis of both acute and chronic liver failure. Timely diagnosis is helpful in averting the progression of liver disease and to stratify the patients presenting with acute liver failure for liver transplantation.

\section{References}

1. Coffey AJ, Durkie M, Hague S, et al. a genetic study of wilsons disease in the united kingdom. Brain 2013;136:1476-87.

2. Gao J, Brackley S, Mann JP. The global prevalence of wilsons disease from next generation sequencing data. Genet Med 2018.

3. Kumar V, Abbas, AK, Aster JC. Robbins Basic pathology. $10^{\text {th }}$ ed. New york, NY: Elsevier, 2018.

4. Roberts EA, Schilsky ML, American association for study of liver diseases (AASLD). Diagnosis and treatment of Wilson disease: an update. Hepatology 2008 june. 47(6):2089-111.

5. Bowcock AM, Farrer LA, Hebert JM, et al. eight closely linked loci place the Wilson disease locus with in 13q14-q21. Am J Hum Genet. 1988 nov. 43(5):664-74.

6. Steindl P, Ferenci P, Dienes HP, Grimm G, Pabinger I, Madl C, et al. wilsons disease in patients presenting with liver disease: a diagnostic challenge. Gastroenterology. 1997,113(1):212-8.

7. Stromeyer FW, Kamal GI, Gerber MA, Mathew T. Groundglass cells in 
hepatocellular carcinoma. Am J

Clinpathol. 1980,74(3): 254-8.

8. Johncilla M, Mitchell KA. Pathology of liver in copper overload. Semin liver disease. 2011,31(3):239-44.

9. Vieira Barbosa joana, Fraga Montserrat, Saldarriaga Joan, Hiroz Phillipe, Giostra Emiliano, Sempoux Christine, Ferenci Peter, Moradpour Darius. Hepatic manifestations of wilsons disease. Swiss medley week.2018:148:w14699. 